\title{
Evaluation of Maternal and Fetal Stress Hormones During the Process of Birth
}

\author{
Burcu KISA KARAKAYA ${ }^{1}$, Ozlem MORALOGLU ${ }^{1}$, Rahime BEDIR FINDIK ${ }^{1}$, Necati HANCERLIOGULLARI ${ }^{1}$ \\ Hatice KANSU CELIK ${ }^{1}$, Tugba CANDAR ${ }^{2}$, Halil Ibrahim YAKUT ${ }^{1}$
}

Ankara, Turkey

\begin{abstract}
OBJECTIVE: This study aims to determine whether mode of delivery is associated with the endocrine stress response in mother and newborn.

STUDY DESIGN: This prospective observational study was conducted with 86 women with a normal singleton pregnancy who delivered healthy infants between 37 and 41 weeks of gestation in a tertiary center. Study groups included; (1) women undergoing normal vaginal delivery with epidural anesthesia, (2) women undergoing vaginal delivery with immersion in water for pain relief during labor, (3) women delivered through elective caesarean section without labor. After delivery, thyroid stimulating hormone, cortisol, insulin, prolactin and Beta-endorphin levels were measured in maternal and umbilical cord serum and their relationships between modes of delivery were investigated.
\end{abstract}

RESULTS: It was found that the concentrations of cortisol and beta-endorphin after vaginal delivery with immersion in water group in both mothers and infants were higher than other two modes of delivery and these differences were statistically significant. Umbilical cord concentration of cortisol was the lowest in the caesarean section group.

CONCLUSIONS: Maternal and fetal stress response was found to be associated with the mode of delivery and labor.

Keywords: Stress hormones, Delivery, Newborn, Immersion in water

Gynecol Obstet Reprod Med 2018;24(2):65-70

\section{Introduction}

Hormonal physiology of childbearing has evolved over millions of years to optimize reproductive success (1). Early prenatal exposure to stress or stress during birth alters the hypothalamic-pituitary adrenal axis and influences further development of the newborn (2). There are four impactful hormonal systems during the birth process: oxytocin; beta-endorphins

1 University of Health Sciences Zekai Tahir Burak Women's Health Training and Research Hospital Obstetrics and Gynecology, Ankara

${ }^{2}$ Ufuk University Medical Faculty of Dr. Rıdvan Ege Applied Health Research Hospital, Ankara

Address of Correspondence: Burcu Kisa Karakaya

Zekai Tahir Burak Women's Health Care, Training and Research Hospital, 06230 Ankara, Turkey

kisaburcu@gmail.com

Submitted for Publication: $\quad$ 15.11.2017

Accepted for Publication:

02.12 .2017

\begin{tabular}{|c|c|}
\hline \multicolumn{2}{|c|}{ Access this article online } \\
\hline 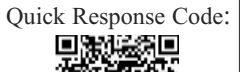 & $\begin{array}{l}\text { Website: www.gorm.com.tr } \\
\text { e-mail: info@gorm.com.tr }\end{array}$ \\
\hline 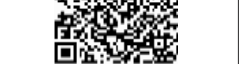 & DOI:10.201613/GORM.2017.753 \\
\hline
\end{tabular}

How to cite this article: Kisa Karakaya B. Moraloglu O. Bedir Findik R. Hancerliogullari N. Kansu Celik H. Candar T. Yakut HI. Evaluation of Maternal and Fetal Stress Hormones During the Process of Birth. Gynecol Obstet Reprod Med 2018;24(2):65-70
( $\beta$-EP); epinephrine-norepinephrine (adrenaline-noradrenaline) and related stress hormone systems; and prolactin (PRL). In addition to its potential role in the onset of human parturition, cortisol constitutes a major component of the endocrinological stress response (1). These hormone systems have complex interactions in the perinatal period, including promoting or inhibiting activity of each other (3). This can amplify hormonal effects, leading to the peaks that characterize physiologic birth. For example, late-labor oxytocin peaks, promoted by high levels of prolactin and oxytocin itself, assist with the pushing stage. Similarly, excessive stress and stress hormones may disrupt labor progress via hormonal interorchestration $(3,4)$. PRL, which regulates feeding and appetite, suppresses ACTH secretion in response to stress (4). Corticosteroids, which are released in case of stress, are used for pulmonary maturation because it increases synthesis of surfactant required in maturation of the lung. The endorphins released during delivery are known to increase prolactin release and this plays a role in this active pulmonary maturation. $\beta$-EP is a natural opioid peptide produced in increased amounts by the body under a wide range of stressful conditions (4). Maternal plasma $\beta$-EP levels rise during labor reaching a maximum at the time of vaginal delivery (5), and possibly serving as an endogenous analgesic for the mother. Several investigators were unable to find any correlation between maternal and cord $\beta$-EP at delivery (5-7). Other researchers detected significant posi- 
tive correlations between maternal and cord or fetal $\beta$-EP (68-9). According to some research, two-day-old infants with lower $\beta$-EP levels had higher amount of neurological soft signs than those with higher levels (1). These hormonally mediated processes are intertwined and continuous with the biologic processes of parturition. Thus, disruption of perinatal hormonal physiology may impact not only labor and birth, but also breastfeeding and maternal-infant attachment (3). On her report, Buckley S. provides powerful evidence for the risks of not letting labor begin on its own (4). Many of the critical hormonal processes preparing both mother and baby for birth occur in the days or even hours before the spontaneous onset of labor (3). This includes increases in the number of hormone receptors, a surge in fetal catecholamines that prepares the fetus for air breathing after birth, and a surge in maternal oxytocin that is transferred to the baby and provides a neuroprotective effect during labor and birth according to some animal studies (2). Previous literature describes a difference in the physiological stress response of children delivered by caesarean section (C-section) as compared with those delivered spontaneously (9-10). According to these studies, the mode of delivery results in specific amounts of cortisol in blood samples taken from the umbilical cord immediately at birth (1014). Present study was designed to assess the relationships between different modes of delivery and hormone responses in both mother and newborn by determining stress-related hormone concentrations in both maternal and umbilical cord plasma.

\section{Material and Method}

\section{Participants}

This prospective open study was conducted at the Delivery Department of Obstetrics of University of Health Sciences Zekai Tahir Burak Women's Health Education \& Research Hospital between January 2014 and June 2015. Exclusion criteria were pre-existing hypertension, pre-eclampsia, pre-existing diabetes mellitus, glucose intolerance, chronic diseases, and premature rupture of fetal membranes and abnormal umbilical Doppler waveforms, antenatal corticosteroid treatment. The study was approved by the local ethic committee of our hospital (2014 \#43), and all women gave written informed consent before enrollment into the study. Groups included (1) women undergoing normal vaginal delivery with epidural anesthesia (NVDE), (2) women undergoing vaginal delivery with immersion in water for pain relief during labor (IW-VD), (3) women who delivered by elective C-section without labor (Elective-planned C-section ELCS). Data were collected regarding all women's age, gestation, parity (nulliparous or multiparous), previous $\mathrm{C}$-section, labor onset (spontaneous or ruptured of membranes) and cervical dilatation, water temperature, duration of pool use, reason for leaving the pool (if she got out prior to delivery), duration of labor and the type of delivery (spontaneous vertex vaginal, C-section).

\section{NVDE Group}

We usually do active management during the active phase (after the cervix dilated to $4-5 \mathrm{~cm}$ ) of labor and use of some interventions in our clinic. The interventions that we use for active management in our standard clinical protocol are amniotomy, low dose I.V. oxytocin infusion (Pitocin- 60-unit oxytocin in $1000 \mathrm{~mL}$ crystalloid (60 miliunits in one $\mathrm{mL}$, an IV infusion - drip method- by using an infusion pump), epidural anesthesia and continuous electronic fetal monitoring. We use a low-dose oxytocin protocol (dose of oxytocin was initiated at 0.5 to 1 miliunits per minute and increased by 1 miliunits per minute at 30 to 40 minute intervals).

\section{IW-VD Group}

Volunteering women with gestational age $>37$ weeks, normal sized fetus, reactive cardiotocogram, clear amniotic fluid and a pregnancy with cephalic presentation were given the choice of immersion in water during labor for pain relief. Exclusion criteria for immersion in water during labor were; intrauterine growth restriction, pathological or suspicious fetal cardiotocogram, meconium stained amniotic fluid, maternal infection with hepatitis B, C, HIV or acute genital herpetic infection, fetal macrosomia. If a subject chose water immersion and there were no contraindications, a soapy water enema was administered and the patient showered before the cervix was dilated to $5 \mathrm{~cm}$. After the cervix dilated to $5 \mathrm{~cm}$, the subject entered in the warm water tub. During water immersion the subject was accompanied by her spouse or significant other and the obstetrician or midwife, and she was encouraged to drink water. Attention was paid to symptoms of dehydration (including rapid heartrate, hidrosis, dizziness, and nausea and vomiting) to avoid circulatory failure. Water temperature was maintained at $35-38^{\circ} \mathrm{C}$.

\section{ELCS Group}

In our study, ELCS group included the women who delivered by $\mathrm{C}$-section in previous birth and have completed at least 38 6/7 weeks of gestation in current pregnancy before onset labor. No other indications are acceptable for elective Csection except for those that already had $\mathrm{C}$-section in previous birth in our hospital setting. Spinal analgesia was used for ELCS Group.

\section{Design and procedures}

Blood samples for determining hormone levels were collected within 60-120 seconds after delivery. We measured hormone levels in an antecubital venous blood sample from the mother and in a venous umbilical cord blood sample collected using a Vacutainer system (Becton Dickinson, Meylan, France). Cord blood was collected from 98 deliveries, yielding 86 complete samples. Other samples were lost or not collected due to hemolysis, collection of only one of the maternal-cord pair, equipment breakdown, lack of project resident coverage during delivery, or delay in processing or freezing the samples. Thyroid stimulating hormone(TSH), Cortisol (CORT), Insulin, Prolactin (PRL) and Beta-endorphin ( $\beta$-EP) were measured by Beckman Coulter Unicell DXI 800 Access Immunoassay Analyzer (Beckman Coulter Ireland, Inc). 
Access TSH assay is a two-site immunoenzymatic (sandwich) assay, for the quantitative determination of human TSH in serum. The intraassay and interassay and imprecision coefficients of variation for TSH were $<2.53 \%$ and $4.55 \%$ respectively. Insulin assay utilizes the 'sandwich principle' with a monoclonal insulin-specific 'capture' antibody and a 'detection antibody labeled with alkaline phosphatase, which is also specific. The intraassay and imprecision coefficients of variation for Insulin were $<2.6 \%$ and $<4.5 \%$, respectively. Access system was used for the other measurements. The intraassay and imprecision coefficients of variation for PRL were $<1.42 \%$ and $<3.32 \%$, respectively. The intraassay and imprecision coefficients of variation for CORT were $<4.4 \%$ and $<6 \%$, respectively. Tuba Human Beta-Endorphin analysis was performed on ELISA-AUORESNESS (Chemwell 2900ALGEN) by Sunredbio ELISA kit. The kit utilizes a doubleantibody sandwich enzyme-linked immunosorbent assay to assay the level of Human Beta-Endorphin ( $\beta$-EP) in samples. The intraassay and interassay coefficients of variation for human-beta endorphin were $<9 \%$ and $<11 \%$ respectively.

\section{Statistical analysis}

Data have been transferred to SPSS 11.5 for Windows (Chicago, INC.) packet program. Statistical analysis was performed by using One-way ANOVA and Bonferroni test, Kruskal-Wallis One-way ANOVA, Mann-Whitney U test, and t-test for independent samples. We also used Pearson Correlation Analysis to detect relationship between maternal and neonatal stress hormones. A p-value of $\leq 0.05$ was considered to be statistically significant.

\section{Results}

Complete data were available for the following mother/ newborn pairs:

28 women delivered vaginally with epidural anesthesia, 28 women delivered vaginally with hydrotherapy for pain relief during labor, 30 women underwent planned- C-section without labor with spinal analgesia.

All 1 minute Apgar scores were $>8$. Participant's characteristics are shown in detail in table 1.

There was no statistically significant difference in the duration of the $1^{\text {st }}, 2^{\text {nd }}$ and the $3^{\text {rd }}$ stages of labor between the (IW-VD) and (NVDE) groups $(p>0.005)$. There was no episiotomy requirement in the groups and the degree of perineal lacerations during birth were not different between the (IWVD) and (NVDE) groups $(p>0.005)$.

\section{Maternal blood hormone concentrations}

Concentrations of CORT and $\beta$-EP in maternal blood were significantly higher after (IW-VD) than after (NVDE) or (ELCS) $(\mathrm{p}=0.001)$. PRL levels were significantly higher after (ELCS) than after all other modes of delivery. Also Insulin levels were significantly lower after (NVDE) than (IW-VD) and (ELCS) $(\mathrm{p}=0.017)$ (Table 2).

Table 1: Patients' characteristics

\begin{tabular}{|c|c|c|c|}
\hline & $\begin{array}{l}\text { IW-VD } \\
(n=28)\end{array}$ & $\begin{array}{l}\text { NVDE } \\
(n=28)\end{array}$ & $\begin{array}{l}\text { ELCS } \\
(n=30)\end{array}$ \\
\hline Age (years) ${ }^{a}$ & $29.1 \pm 5.8$ & $27.18 \pm 5.5$ & $30.9 \pm 6$ \\
\hline Parity ${ }^{b}$ & $1(1-3)$ & $1(1-5)$ & $1(1-4)$ \\
\hline BMI $\left(\mathrm{kg} / \mathrm{m}^{2}\right)^{\mathrm{a}}$ & $28.3 \pm 3.9$ & $30.2 \pm 4.1^{*}$ & $26.4 \pm 4$ \\
\hline Birthweight $(g)^{a}$ & $3406.9 \pm 391.3$ & $3330 \pm 406.3$ & $3278.2 \pm 419.3$ \\
\hline Apgar's score $1 \mathrm{~min}^{\mathrm{b}}$ & $9.5(8-10)$ & $9(8-10)$ & $9(7-10)$ \\
\hline
\end{tabular}

BMI: Body mass index, IW-VD: Vaginal delivery with immersion water for pain relief during labor, NVDE: Normal vaginal delivery with epidural anesthesia, ELCS: Elective C-section without labor, a: Values are given as mean $\pm S D,{ }^{b}:$ Values are given as median(range), *Significant difference $(p=0.003)$ between NVDE and ELCS by One-way ANOVA.

Table 2: Maternal hormone concentrations and mode of delivery

\begin{tabular}{|c|c|c|c|}
\hline & $\begin{array}{c}\text { IW-VD } \\
(n=28) \\
\text { Mean } \pm S D\end{array}$ & $\begin{array}{c}\text { NVDE } \\
(n=28) \\
\text { Mean } \pm S D\end{array}$ & $\begin{array}{c}\text { ELCS } \\
(n=30) \\
\text { Mean } \pm S D\end{array}$ \\
\hline TSH (mlU/mL) & $2.37 \pm 0.2$ & $2.86 \pm 0.27$ & $2.58 \pm 0.28$ \\
\hline Insulin(mLU/mIL) & $9.86 \pm 2.26$ & $4.79 \pm 0.81^{*}$ & $8.80 \pm 2.04$ \\
\hline $\mathrm{PRL}(\mathrm{ng} / \mathrm{mL})$ & $145.5 \pm 9.7$ & $148.3 \pm 7.5$ & $167.2 \pm 8.3^{* *}$ \\
\hline CORT ( $\mu \mathrm{g} / \mathrm{dL})$ & $57.4 \pm 3.6^{* * *}$ & $47.9 \pm 2.18$ & $21.37 \pm 2.1$ \\
\hline$\beta-E P(n g / L)$ & $286.4 \pm 40^{\star * *}$ & $115 \pm 22.6^{*}$ & $167 \pm 26.4$ \\
\hline
\end{tabular}

TSH: Thyroid stimulant hormone, PRL: Prolactin, CORT: Cortisol, $\beta$-EP: Beta endorphin, IW-VD: Vaginal delivery with immersion water for pain relief during labor, NVDE: Normal vaginal delivery with epidural anesthesia, ELCS: Elective $C$-section without labor, *Significant difference ( $p=0.017)$ between normal NVDE and all other modes of delivery, ${ }^{* *}$ Significant difference $(p=0.001)$ between ELCS and all other modes of delivery, ${ }^{* * *}$ Significant difference $(p=0.001)$ between IW-VD and all other modes of delivery 


\section{Umbilical cord blood hormone concentrations}

Umbilical cord concentration of TSH was highest $(10.6 \pm 1.54 \mathrm{mLU} / \mathrm{mL}, p=0.017)$ in (NVDE) group, in contrast Insulin level was the lowest $(4.79 \pm 0.81 \mathrm{mLU} / \mathrm{mL}, p=0.017)$ (Table 3). Umbilical cord concentrations of CORT and $\beta$-EP were significantly higher in (IW-VD) group than in all other modes of delivery $(23.9 \pm 3.65 \mu \mathrm{g} / \mathrm{dL}, 320 \pm 48.6 \mathrm{ng} / \mathrm{l}, p=0.001)$ (Table 3). There was a linear correlation between maternal serum and umbilical cord concentrations of $\beta$-EP which was statistically significant $(\mathrm{r}=0,828, p=0,001)$ (Figure 1). We could not find any correlation between maternal serum and umbilical cord concentrations of CORT to be statistically significant $(\mathrm{r}=-0,192, p=0,336)$.

\section{Discussion}

The physiologic process of labor and birth is largely driven by hormones, and the hormonal orchestration of the process is easily disrupted. Buckley provides a seminal systematic review of this complex interplay of hormones that prepare the body for birth and then orchestrate the process of labor (4). Common maternity care practices and interventions can impact the hormonal physiology of mother and baby, according to physiologic understandings and human and animal studies (3). We investigated the relationship between the mode of delivery and hormone concentrations in both mother and infant in a large sample. We found the highest concentrations of CORT and $\beta$-EP after (IW-VD) group in both mothers and infants than after the two modes of delivery and these differences were statistically significant. Gitau et al. and Mears et al. found that fetal CORT concentrations were reduced in newborns delivered by $\mathrm{C}$-section when compared to vaginal delivery, whereas assisted vaginal delivery resulted in elevated CORT levels when compared with normal vaginal delivery $(9,10)$. Vogl et al. compared hormone concentrations in both mother and child in different modes of delivery in their study (11). They reported that concentrations of epinephrine (EP), nor-epinephrine (NOR), adrenocorticotrophic hormone (ACTH), CORT and $\beta$-EP in maternal blood were significantly lower after elective $\mathrm{C}$-section than after unassisted normal vaginal delivery without pain relief, vaginal delivery with epidural anesthesia or delivery by ventouse extraction (11). They found that only PRL levels were significantly higher after elective $\mathrm{C}$-section than after all other modes of delivery. PRL levels were significantly higher in maternal serum after elective $\mathrm{C}$-section than after all other modes of delivery in our study similarly to Vogl's study (11). We found that umbilical cord concentrations of CORT and $\beta$-EP were significantly higher in (IW-VD) group than all other modes of delivery and especially umbilical cord concentration of CORT was the lowest in the elective C-section group. Similarly, Vogl et al. reported that umbilical cord concentration of CORT was significantly lower in the elective $\mathrm{C}$-section group than in all other modes of delivery (11). Otherwise, they concluded that $\beta$-EP concentrations in newborns after epidural anesthesia were also significantly higher than after vaginal delivery without pain relief, elective $\mathrm{C}$-section or ventouse extraction.

Gülmezoglu et al. assessed induction of labor compared with waiting for spontaneous labor (expectant management) in women with pregnancies at or beyond term (15). They concluded that induction of labor to avoid prolonged pregnancy may be associated with fewer perinatal deaths than expectant management. During induction of labor, health care professionals should provide women with the pain relief appropriate for them and their pain. This can range from simple analgesics to epidural analgesia (16). Epidural and spinal analgesic techniques provide optimal pain relief for parturient (17). Although they are generally quite safe, these techniques are also associated with various untoward effects. Relieving pain during childbirth represents an important challenge for both health care professionals and pregnant women (16-20). Pain relief strategies include non-pharmacologic and pharmacologic approaches (20-25). In obstetrics, pharmacologic methods such as epidural anesthesia have proven to be efficient in reducing pain during labor and are now routinely used, and even expected, to manage pain (18). Some authors have suggested that this process may contribute to an over-medicalization of women's child birth experiences (18). Non-pharmacologic approaches to relieve pain during labor, when used as a part of hospital pain relief strategies, provide significant benefits to women and their infants without causing additional harm. Non-pharmacological methods of labor analgesia in-

Table 3: Cord blood hormone concentrations and mode of delivery

\begin{tabular}{lccc}
\hline & $\begin{array}{c}\text { IW-VD } \\
(\mathrm{n}=28) \\
\text { Mean } \pm \text { SD }\end{array}$ & $\begin{array}{c}\text { NVDE } \\
(\mathrm{n}=28) \\
\text { Mean } \pm S D\end{array}$ & $\begin{array}{c}\text { ELCS } \\
(\mathrm{n}=30) \\
\text { Mean } \pm S D\end{array}$ \\
\hline TSH $(\mathrm{mlU} / \mathrm{mL})$ & $6.2 \pm 0.72$ & $10.6 \pm 1.54^{*}$ & $5.8 \pm 0.42$ \\
$\operatorname{lnsulin}(\mathrm{mlU} / \mathrm{mL})$ & $9.86 \pm 2.26$ & $4.79 \pm 0.81^{*}$ & $8.8 \pm 2.04$ \\
$\mathrm{PRL}(\mathrm{ng} / \mathrm{mL})$ & $188 \pm 6.36$ & $187 \pm 6.7$ & $190 \pm 6.87$ \\
$\operatorname{CORT}(\mu \mathrm{g} / \mathrm{dL})$ & $23.9 \pm 3.65^{* *}$ & $14.8 \pm 1.7$ & $6.7 \pm 0.87$ \\
$\beta$-EP $(\mathrm{ng} / \mathrm{L})$ & $320 \pm 48.6^{* *}$ & $120 \pm 21.9^{*}$ & $157 \pm 34$ \\
\hline
\end{tabular}

TSH: Thyroid stimulant hormone, PRL: Prolactin, CORT: Cortisol, $\beta$-EP-Beta endorphin, IW-VD: Vaginal delivery with immersion water for pain relief during labor, NVDE: Normal vaginal delivery with epidural anesthesia, ELCS: Elective C-section without labor, * Significant difference ( $p=0,017)$ between NVDE and all other modes of delivery, ${ }^{* *}$ Significant difference $(p=0.001)$ between IW-VD and all other modes of delivery 
clude doula support during labor and delivery, music therapy, postural changes, water immersion and birth, and acupuncture (20-24). A Cochrane systematic review of immersion in water in labor and birth found that immersion in water significantly reduced the duration of the first stage of labor and reduced the use of epidural anesthesia, but did not significantly reduce intrapartum C-section in the included RCTs (25). During labor, pain plays an important role in the production of natural pain relief hormones, such as endogenous oxytocin and endorphins, which also contribute to regulate uterine contractions (26-34). If the woman requires an epidural and oxytocin augmentation, she does not experience this endorphin release because exogenous oxytocin (Pitocin) does not cross the bloodbrain barrier (29-36). Similarly, we found highest concentrations of serum $\beta$-EP after (IW-VD) without intervention group in both mothers and their infants than after the two modes of delivery and there was a linear correlation between mother's and newborn's $\beta$-EP concentrations. Also, we found lowest concentrations of serum Insulin and $\beta$-EP after NVDE (intervention) group in both mothers and their infants than after the two modes of delivery. We could not find any correlation between maternal serum and umbilical cord concentrations of CORT similarly to Mears et al (10). Their data suggested that maternal CORT levels contribute little to the fetal CORT concentrations, as has been believed previously.

Miller et al. (12) reported in their study that, intrapartum C-section group did have increased cortisol levels, and this correlated positively with the amount of labor experienced, as determined by cervical dilatation at the time of C-section. Due to the performance of elective $\mathrm{C}$-section prior to onset of labor in our setting, we could not include another group with C-section after onset of labor. Nevertheless, this question should eventually be addressed in future studies.

\section{Conclusion}

Our study reports variances in hormonal levels of the mother and the newborn depending on the method of delivery. Whether the limited stress reaction and reduced stress hormone responses seen after delivery by elective $\mathrm{C}$-section have a beneficial or a deleterious impact on the child's later neurocognitive and motor development $(2,7,12-14)$. It may be considered part of the process of fetal programming of the HPA with all its potential health hazards for the affected child. Research priorities include better understanding of many aspects of hormonal physiology and of impacts of maternity interventions on breastfeeding, maternal adaptations, maternal mood, and other short, medium, and long term hormonally mediated and developmental outcomes $(3,31)$.

\section{: Acknowledgments: None}

Conflict of interest: The authors reported no conflict of interest.

\section{References}

1. Amis D. A Childbirth Educator's Commentary on Hormonal Physiology of Childbearing: Evidence and Implications for Women, Babies, and Maternity Care. J Perinat Educ 2015;24(3):154-9.

2. Rothenberg SJ, Chicz-DeMet A, Schnaas L, Karchmer S, Salinas V, Guzmán LA. Umbilical cord beta-endorphin and early childhood motor development. Early Hum Dev 1996;46(1-2):83-9.

3. Buckley SJ. Executive summary of hormonal physiology of childbearing: Evidence and implications for women, babies, and maternity care. J Perinat Educ 2015;24(3): 145-53.

4. Buckley SJ. Hormonal physiology of childbearing: Evidence and implications for women, babies, and maternity care. Washington, DC: Childbirth Connections Programs, National Partnership for Women \& Families; 2015; 98-100.

5. Grattan DR, Pi XP, Andrews ZB, Augustine RA, Kokay IC, Summerfield MR. et al. Prolactin receptors in the brain during pregnancy and lactation: implications for behaviour. Horm Behav 2001;40(2):115-24.

6. Zivný J, Kobilková J, Vorlicek F, Bendl J, Zapadlo M. Plasma beta-endorphin-like immunoreactivity during pregnancy, parturition, puerperium and in newborn. Acta Obstet Gynecol Scand 1986;65(2):129-31.

7. Chan EC, Smith R, Lewin T, Brinsmead MW, Zhang HP, Cubis J. et al. Plasma corticotropin-releasing hormone, beta-endorphin and cortisol inter-relationships during human pregnancy. Acta Endocrinol 1993;128(4):339-44.

8. Radunovic N, Lockwood, CJ, Alvarez M, Nastic D, Berkowitz RL. Beta-endorphin concentrations in fetal blood during the second half of pregnancy. Am J Obstet Gynecol 1992;167(3):740-4.

9. Gitau R, Menson E, Pickles V, Fisk NM, Glover V, MacLachlan N. Umbilical cortisol levels as an indicator of the fetal stress response to assisted vaginal delivery. Eur J Obstet Gynecol Reprod Biol 2001;98(1):14-7.

10. Mears K, McAuliffe F, Grimes H, Morrison JJ. Fetal cortisol in relation to labour, intrapartum events and mode of delivery. J Obstet Gynaecol 2004;24(2):129-32.

11. Vogl SE, Worda C, Egarter C, Bieglmayer C, Szekeres T, Huber J. et al. Mode of delivery is associated with maternal and fetal endocrine stress response. BJOG 2006; 113(4):441-5.

12. Miller NM, Fisk NM, Modi N, Glover V. Stress responses at birth: determinants of cord arterial cortisol and links with cortisol response in infancy. BJOG 2005;112(7): 921-6.

13. Bird JA, Spencer JA, Mould T, Symonds ME. Endocrine and metabolic adaptation following caesarean section or vaginal delivery. Arch Dis Child Fetal Neonatal Ed 1996; 
74(2):F132-4

14. Charmandari E, Kino T, Souvatzoglou E, Chrousos GP. Pediatric stress: hormonal mediators and human development. Horm Res 2003;59(4):161-79.

15. Gulmezoglu AM, Crowther CA, Middleton P, Heatley E. Induction of labour for improving birth outcomes for women at or beyond term. Cochrane Database Syst Rev 2012(6):CD004945.

16. Kannan S, Jamison RN, Datta S. Maternal satisfaction and pain control in women electing natural childbirth. Reg Anesth Pain Med 2001;26(5):468-72.

17. Ellen DH. Pain and women's satisfaction with the experience of childbirth: A systematic review. Am J Obstet Gynecol 2002;186(15):160-72.

18. Anim-Somuah M, Smyth R, Howell C. Epidural versus non-epidural or no analgesia in labour. Cochrane Database Syst Rev 2011(12):CD000331.

19. Brownridge P. Treatment options for the relief of pain during childbirth. Drugs 1991;41(1):69-80.

20. Chaillet N, Belaid L, Crochetiere C, Roy L, Gagne GP, Moutquin JM. et al. Non pharmacologic approaches for pain management during labour compared with usual care: A meta-analysis. Birth 2014;41(2):122-37.

21. Smith CA, Collins CT, Cyna AM, Crowther CA. Complementary and alternative therapies for pain management in labour. Cochrane Database Syst Rev 2006(4): CD003521.

22. Bergström M, Kieler H, Waldenström U. Effects of natural childbirth preparation versus standard antenatal education on epidural rates, experience of childbirth and parental stress in mothers and fathers: A randomised controlled multicentre trial. BJOG 2009;116(9):1167-76.

23. Batten M, Stevenson E, Zimmermann D, Isaacs C. Implementation of a hydrotherapy protocol to improve postpartum pain management. J Midwifery Womens Health 2017;62(2):210-4.

24. Eckert K, Turnbull D, MacLennan A. Warm water bathing did not reduce use of pharmacologic analgesia during the first stage of labour. Evidence-Based Med 2001;6(6):177.

25. Cluett ER, Burns E: Immersion in water in labour and birth. Cochrane Database Syst Rev. Cochrane Database Syst Rev 2009 (2):CD000111.

26. Leap N, Dodwell M, Newburn M. Working with pain in labour: An overview of evidence. New Digest 2010; 49:22-6.

27. Jouppila R, Jouppila P, Karlqvist K, Kaukoranta P, Leppäluoto J, Vuolteenaho O. Maternal and umbilical venous plasma immunoreactive beta-endorphin levels during labour with and without epidural analgesia. Am J Obstet Gynecol 1983;147(7):799-802.

28. Brinsmead M, Smith R, Singh B, Lewin T, Owens P. Peripartum concentrations of beta endorphin and cortisol and maternal mood states. Aust N Z J Obstet Gynaecol 1985;25(3):194-7.

29. Fuchs AR, Fuchs F, Husslein P, Soloff MS, Fernström MJ. Oxytocin receptors and human parturition: A dual role for oxytocin in the initiation of labour. Science 1982;215 (4538):1396-8.

30. Fuchs AR, Fuchs F, Husslein P, Soloff MS. Oxytocin receptors in the human uterus during pregnancy and parturition. Am J Obstet Gynecol 1984;150(6):734-41.

31. Buckley SJ. Ecstatic Birth: Nature's Hormonal Blueprint for Labour (Internet). 2002 (updated 2010; cited March 2017). Available from: http://sarahbuckley.com/articles

32. Schmid V, Donna S. Birth Pain Power to Transform! a guide for pregnant women. 1st edition: United Kingdom. Fresh Heart Publishing 2011:15-8.

33. Grajeda R, Perez-Escamilla R. Stress during labor and delivery is associated with delayed onset of lactation among urban Guatemalan women. J Nutr 2002;132(10):3055-60.

34. Schuller C, Känel N, Müller O, Kind AB, Tinner EM, Hösli I. et al. Stress and pain response of neonates after spontaneous birth and vacuum-assisted and cesarean delivery. Am J Obstet Gynecol 2012;207(5):415.e1-6.

35. Lothian JA. Healthy Birth Practice: Avoid interventions unless they are medically necessary. J Perinat Educ 2014;23(4):198-206.

36. Bohn MC, Lauder JM. Cerebellar granule cell genesis in the hydrocortisone-treated rats. Dev Neurosci 1980;3 (2):81-9. 